This document is published in:

IEEE Communications Magazine, October 2013, 51(10), 172 - 181.

Doi: 10.1109/MCOM.2013.6619581

(C) 2013 IEEE. Personal use of this material is permitted. Permission from IEEE must be obtained for all other uses, in any current or future media, including reprinting/republishing this material for advertising or promotional purposes, creating new collective works, for resale or redistribution to servers or lists, or reuse of any copyrighted component of this work in other works. 


\title{
On providing mobility management in WOBANs: Integration with PMIPv6 and MIH
}

\author{
M. Isabel Sanchez* ${ }^{* \dagger}$, Manuel Urueña ${ }^{\dagger}$, Antonio de la Oliva $^{\dagger}$, Jose Alberto Hernandez ${ }^{\dagger}$ and Carlos J. Bernardos ${ }^{\dagger}$ \\ *Institute IMDEA Networks, Spain \\ Email: mariaisabel.sanchez@imdea.org \\ ${ }^{\dagger}$ Departamento de Ingenieria Telematica, Universidad Carlos III de Madrid, Spain \\ Email: \{muruenya, aoliva, jahgutie, cjbc\}@it.uc3m.es
}

\begin{abstract}
The Wireless-Optical Broadband Access Network (WOBAN) is a promising access architecture that combines the high performance of optical networks with the ubiquity and convenience of wireless technologies. This article proposes a networkbased mobility framework that is specially tailored for WOBANs. The proposed architecture is based on Proxy Mobile IPv6 and IEEE 802.21 mobility management protocols, but it also defines a number of optimizations that enable the seamless handover of mobile nodes. In particular, the hierarchical architecture together with the broadcast-and-select nature of the optical part of the WOBAN are leveraged to: $i$ ) optimize the mobility of users with respect to the overall network resources, both at the wireless access and optical distribution parts, ii) remove the overhead of IP-in-IP tunneling between the PMIPv6 entities, and iii) perform an efficient bicasting during the handover process to minimize packet loss.
\end{abstract}

Index Terms-Wireless-Optical Broadband Access Network (WOBAN); Ethernet Passive Optical Network (EPON); Proxy Mobile IPv6 (PMIPv6); IEEE 802.21 Media Independent Handover (MIH) Services.

\section{INTRODUCTION AND MOTIVATION}

Recently, the research community has proposed the combination of optical and wireless technologies to provide anywhere-anytime broadband access networks meeting the bandwidth requirements of the next-generation applications. The acronym WOBAN, which stands for Wireless-Optical Broadband Access Networks, has been used by the authors in [1] to refer to a Passive Optical Network (PON) whose termination points are attached to wireless (either WiFi- or WiMAX-based) or cellular access technologies.

WOBANs are seen as attractive broadband access networks since they combine the benefits of PONs:

- high bandwidth capacity, typically 1 - $10 \mathrm{Gbit} / \mathrm{s}$,

- network based on a passive infrastructure that eases Operation, Administration and Management (OAM),

- Capital Expenditure (CAPEX) savings since many users share the optical fiber connection to the Central Office,

- simple interoperability for the case of Ethernet-based PONs,

together with the advantages of mature wireless/cellular technologies:

- radio coverage of hundreds of meters for $\mathrm{WiFi}$, and several kilometers for cellular technologies,

- tens to hundreds of Mbit/s for WiFi, and tens of Mbit/s for cellular technologies,
- wide popularity and availability of wireless devices among end-users.

However, before such a hybrid broadband access network becomes a reality, it is required to study certain aspects of the mobility management of users moving between wireless Access Points (APs) attached to the PON. These, and other related challenges are outlined in [2]. Indeed, previous studies have already addressed a number of key aspects to achieve real optical-wireless integration, i.e. those related to keeping Quality of Service (QoS) over the EPON and WiMAX [3] [4], effective routing strategies considering capacity and delay aspects [5] or from an energy efficiency point of view [6]. However, very few works have considered mobility in WOBANs. For instance, [7] proposes a new, non-standard, mobility mechanism that requires special hardware at the ONUs and PON splitter, as well as support from the end-user mobile node. The authors in [8] also propose to integrate PMIPv6 over EPONs defining two possible architectures, but it requires additional changes to provide mobility support.

This article proposes an integrated mobility architecture for converged optical-wireless architectures, and particularly for WOBANs, based on Proxy Mobile IPv6 (PMIPv6) and the handover optimizations enabled by the use of the IEEE 802.21 Media Independent Handover (MIH) Services. Essentially, the architecture locates the Mobile Access Gateways (MAGs) defined in PMIPv6 at the leaf nodes of the PON, that is, the Optical Network Units (ONUs), and the Local Mobility Anchor (LMA) at the root, i.e. the Optical Line Terminal (OLT). This architecture facilitates the optical-wireless integration for several reasons: First, the OLT-LMA module is able to combine traffic statistics of the ONU-MAGs and mobility information of the end Mobile Nodes (MNs) connected to every MAG. This might be useful to redefine the Dynamic Bandwidth Allocation (DBA) strategies based on the MNs attached to the ONUs, rather than on the ONUs solely. Second, the mobility procedures of PMIPv6 may be optimized thanks to the single-IP-hop, point-to-multipoint topology of the PON, which provides multicast services at no extra bandwidth cost. And third, the OLT-LMA leverages the static location of the ONUs to infer possible destinations of Mobile Nodes, and to design an effective procedure to reduce packet loss during the handover process.

The remainder of this work is thus organized as follows: Section II reviews the architecture of a typical WOBAN and 


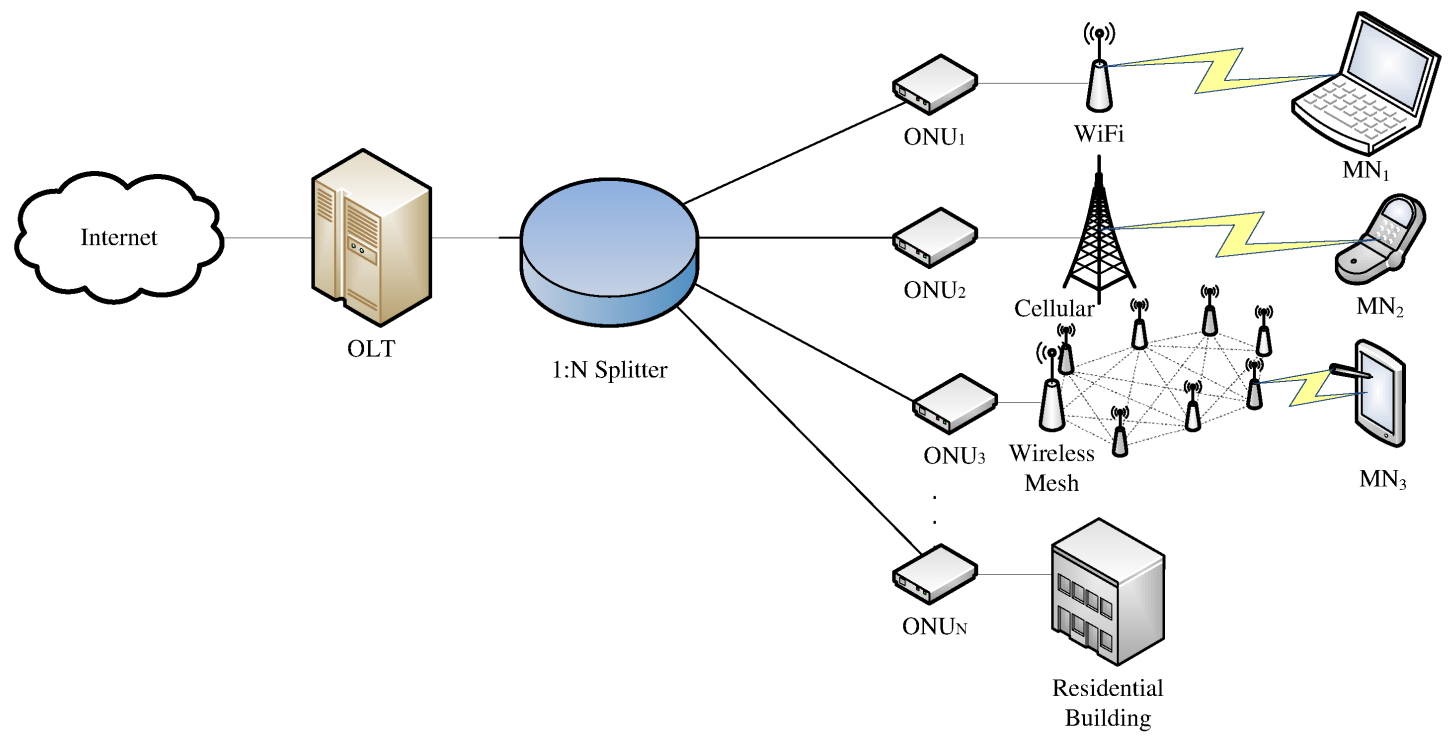

Fig. 1. Ethernet Passive Optical Network (EPON) architecture

the IP mobility management protocol of choice, PMIPv6, together with the technology used to optimize the handover process, IEEE 802.21 Media Independent Handovers (MIH). Section III proposes the integrated PMIPv6-WOBAN architecture that manages end-user mobility across the wireless access points hanging from the ONUs. This section also overviews the benefits of such an integrated architecture, especially concerning seamless handovers, which are further discussed in section IV, including a simulation-based validation of our proposal. Finally, section $\mathrm{V}$ reviews the main contributions of this work.

\section{TECHNOLOGIES INVOLVED}

This section overviews the optical part of a WOBAN, the Proxy Mobile IPv6 mobility-management protocol and the IEEE 802.21 MIH specification, being all of them key technologies to enable mobility in WOBANs.

\section{A. Ethernet Passive Optical Network}

Essentially, a WOBAN is a Passive Optical Network (PON) whose terminating points, the Optical Network Units (ONUs), are attached to one or more heterogeneous wireless Access Points (WiFi, WiMAX, cellular or other). The PON is a passive point-to-multipoint (PtMP) optical access network following a tree topology, as shown in Fig. 1. The leaf nodes, or ONUs, are connected to the root node, referred to as the Optical Line Terminal (OLT) via a passive splitter/combiner that needs no power supply or configuration. The role of such a passive device is two-fold: First, it splits the OLT signal into, typically, 32 or 64 copies for the ONUs in the downstream direction; and second, it combines the signals generated by the ONUs into a single one in the upstream direction. Thus, the PON operates as a broadcast-and-select network in the downstream direction, since the data sourced at the OLT is replicated by the passive splitter/combiner and delivered at all ONUs.
An Ethernet PON (EPON) is a type of PON employing IEEE 802.3/Ethernet frames, which contain a small EPONspecific header in its Preamble (see Fig. 2). The Logical Link Identifier (LLID) field specifies the recipient ONU(s) of the EPON frame, and the Mode bit specifies whether the LLID is unicast $(M=0)$ or broadcast/multicast $(M=1)$. A unicast LLID is assigned to each ONU by the OLT. Hence, upon the reception of an EPON frame, every ONU must check the LLID field to filter out all the frames intended for other ONUs.

On the other hand, the upstream wavelength is shared by all ONUs on a Time Division Multiplexing (TDM) basis, so a channel access arbitration mechanism must be defined to avoid collisions at the passive splitter/combiner. In light of this, the IEEE 802.3ah standard also defines the Multi-Point Control Protocol (MPCP), where the OLT schedules transmission windows to the ONUs after a clock synchronization process. A number of Dynamic Bandwidth Allocation (DBA) algorithms have already been defined in the literature, being Interleaved Polling with Adaptive Cycle Time (IPACT) [9] the most popular one.

In the following, we consider that the PON is terminated either on single wireless Access Points or any other wireless layer-2 cloud (for instance, wireless mesh topologies), as long as these behave as a single IP hop. Next, we review PMIPv6 and MIH, two key protocols to enable user mobility in WOBANs.

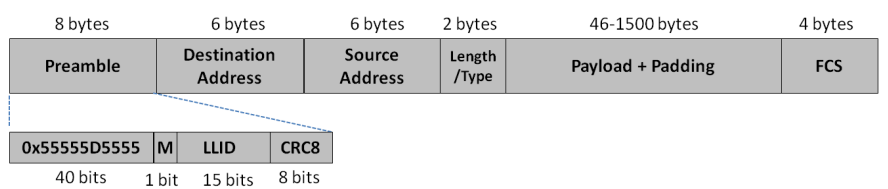

Fig. 2. IEEE 802.3ah EPON frame format 


\section{B. Proxy Mobile IPv6}

Proxy Mobile IPv6 (PMIPv6) is a mobility-management protocol that allows legacy mobile terminals to perform handover operations across heterogeneous networks, without their involvement in the management of their own IP mobility signaling. As an example of operation (see Fig.3), consider a Localized Mobility Domain (LMD) scenario, where PMIPv6 provides mobility support, that comprises two Mobile Access Gateways (MAGs), and a Local Mobility Anchor (LMA). In addition to maintaining the state regarding the location of the MN in the LMD, the LMA must maintain an IPv6-in-IPv6 tunnel with every MAG for forwarding the data traffic of their MNs. When a MN first arrives at the LMD, it attaches to an Access Point (AP) and sends a Router Solicitation (RS) message requesting an IPv6 prefix. This message is received by the MAG, which asks the LMA for an IPv6 prefix for the MN through a Proxy Binding Update message. Next, the LMA replies to the MAG with a newly assigned IPv6 prefix for the MN through a Proxy Binding Acknowledgment message and stores the mapping in its local lookup table, named Binding Cache. Then, the MAG forwards the IPv6 prefix to the MN through a Router Advertisement (RA) message. Finally, the LMA uses the existing IPv6-in-IPv6 tunnel with the MAG (or creates a new one if there is none) for the data traffic exchanged by the MN with the network. When the MN moves to the coverage area of a second MAG, the process is repeated, but this time the LMA finds an existing entry in its Binding Cache for that $\mathrm{MN}$, and therefore replies to the MAG with the same IPv6 prefix that the MN was using previously, updating the record for the $\mathrm{MN}$ and diverting its traffic to the new MAG tunnel. Thanks to the fact that the MAGs show the same layer2 and IPv6 link local addresses to the MNs, these do not detect any layer-3 change while moving within the LMD.

In conclusion, thanks to PMIPv6, a Mobile Node may change from one layer-2 Point of Attachment (PoA) to another, but it always keeps the same IP address across the LMD managed by an LMA. It is also worth noticing that the operation of PMIPv6 does not require the MN to implement any modification or extra software in its layer-3 stack, although it may require the assistance of some layer- 2 mechanisms to work more efficiently. These mechanisms are known as linklayer triggers, and are required to quickly detect a change of layer-2 PoA. In the proposed architecture we rely on IEEE 802.21 MIH to provide such link-layer trigger functionality, as described next.

\section{IEEE 802.21 Media Independent Handover Services}

The IEEE 802.21 Media Independent Handover (MIH) Services [10] standard defines a common interface to allow the optimization of handovers between heterogeneous IEEE 802 systems, as well as between IEEE 802 and cellular systems. This is achieved by adding a technology-independent function - the Media Independent Handover Function (MIHF) - that improves the communication between different entities, either locally (mobile node) or remotely (network functions).

The MIH standard defines different roles according to the relationship between the network-based MIHFs and the MN.

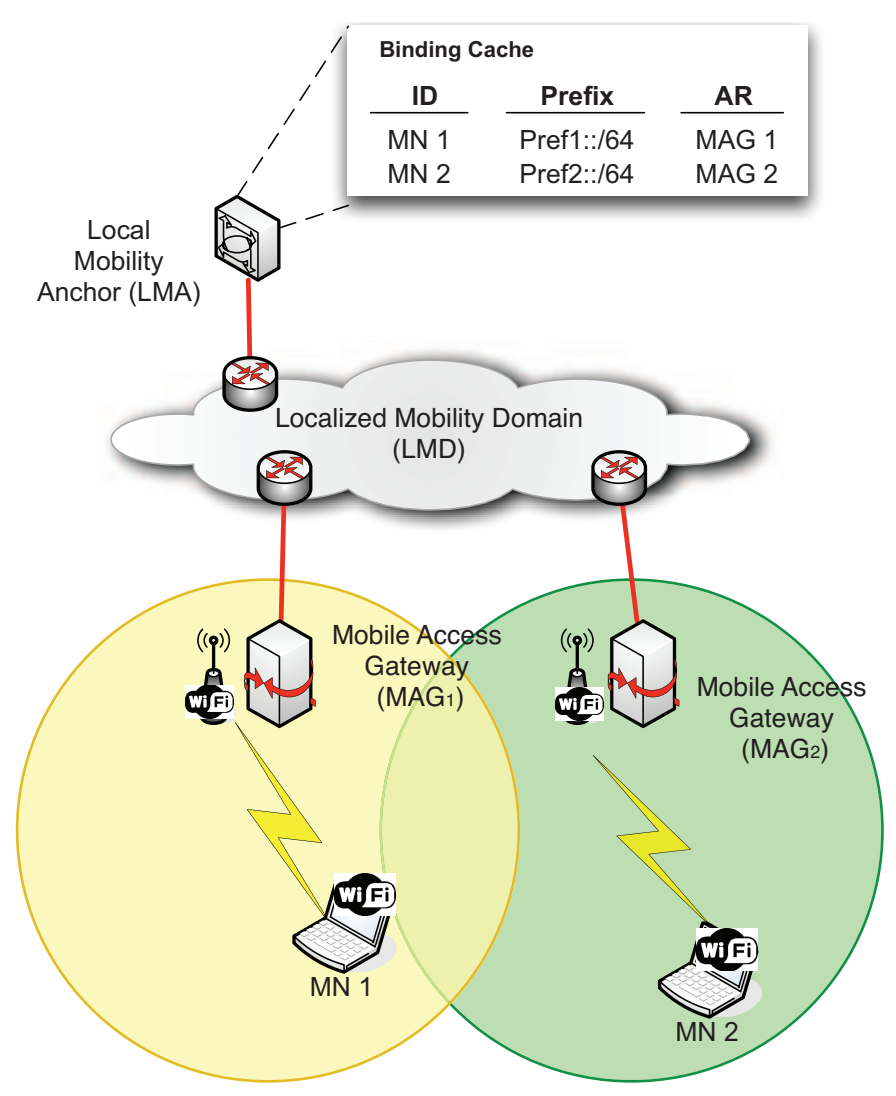

Fig. 3. Proxy Mobile IPv6 (PMIPv6) scenario

These are the Point of Service (PoS) and Point of Attachment (PoA). The former identifies a network-based MIHF that talks directly with an $\mathrm{MN}$, while the later corresponds to the network-side end-point of a layer-2 link with the MN.

In addition, MIH defines three main mobility services:

- The Media Independent Event Service (MIES) provides event reporting of dynamic changes in link characteristics, status and quality.

- The Media Independent Command Service (MICS) enables MIH clients to manage and control the link behavior related to handover and mobility.

- The Media Independent Information Service (MIIS) provides details about the characteristics and services provided by the serving and surrounding networks.

The use of MIH in the PMIPv6-WOBAN architecture proposed in the next section is twofold: On the one hand, the link-layer event support provided by the MIES is employed to trigger certain actions at the MAG, for example sending a Proxy Binding Update upon MN attachment to a connected Access Point. On the other hand, the handover optimization signaling is used to piggyback information that enables the WOBAN mobility optimizations described in the next section.

\section{INTEGRATED PMIPv6-WOBAN ARCHITECTURE}

Fig. 4 (Top) shows the proposed PMIPv6-WOBAN integrated architecture, together with the signaling (left boxes) 


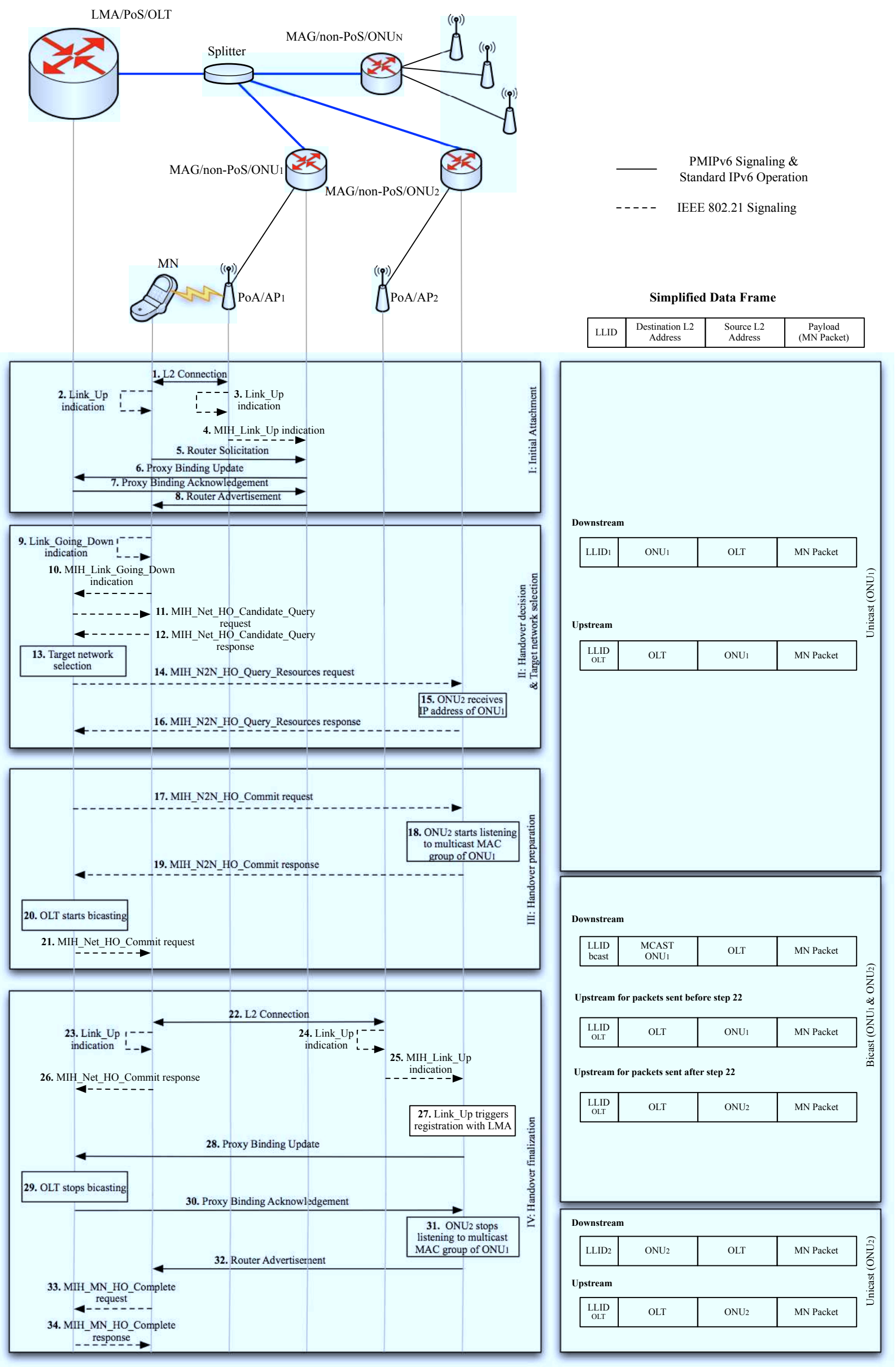

Fig. 4. End-user mobility inside PMIPv6-WOBAN. Signaling on the left boxes, and simplified data frames on the right hand side 
and data (right boxes) paths. For simplicity, we show three ONUs, two of them connected to a single WiFi Access Point, and another one connected to three Access Points. Note that the Localized Mobility Domain comprises the whole radio coverage of the five APs of the WOBAN.

It is worth emphasizing that the PMIPv6-WOBAN architecture requires that any wireless access network connected to an ONU behaves as a single layer-2 domain. In such a case, there is no difference between an isolated AP connected to the MAG or a group of APs forming a mesh, as long as they operate as a single IP hop. The interested reader is referred to the IEEE 802.11s amendment, which details how to deploy a layer-2 wireless mesh.

An EPON exhibits a hierarchical architecture where the OLT dynamically assigns time-slots to the ONUs following some DBA algorithm, for instance IPACT. This structure resembles the one of a PMIPv6-enabled LMD, in which the LMA anchors the IP addresses assigned to the Mobile Nodes that are attached to the MAGs, which are likewise controlled by the LMA. Based on this observation, we propose an integrated PMIPv6-WOBAN architecture, where the LMA is collocated with the OLT, and the MAGs are collocated with the ONUs (one MAG per ONU). From an MIH functionality perspective, we consider that the Point of Service $(\mathrm{PoS})$ resides at the OLT, while all ONUs are also IEEE 802.21 capable, that is, they implement an MIHF acting as non-PoS MIH entities.

It should be noted that, although WiFi has been assumed as the access technology throughout the previous description of the proposed PMIPv6-WOBAN architecture, other technologies are also supported, as long as the mobile node supports IPv6 and IEEE 802.21. Both protocols are defined to operate over different wireless technologies, such as WiMAX and 3 GPP ones, among others. Since ONU-MAG devices behave as IPv6 routers, their interfaces may employ completely different layer-2 technologies, including wireless ones. If the wireless attachment point is not co-located with the ONU but it is a remote device connected by Ethernet to the ONU-MAG, then the wireless technology should support IEEE 802.1D bridging capabilities (so wireless frames could be sent back and forth as Ethernet frames, as well as to support intra-ONU mobility) or the communication between the MAG and the MN should be able to behave as a point-to-point link as stated by PMIPv6. As an example, interworking with 3GPP cellular networks is enabled by the use of PMIPv6, which is one of the solutions adopted by the 3GPP Evolved Packet System (EPS) to provide inter-access mobility support between 3GPP and non-3GPP access networks.

The proposed integrated PMIPv6-WOBAN architecture enables a set of optimizations that enhance the operation of each protocol with the intrinsic characteristics of the other. Next we present these optimizations and describe the architecture by detailing the different mobility-related operations.

\section{A. Initialization and Mobile Node attachment}

The first box of Fig. 4 describes the MIH events (dashed arrows) and PMIPv6 messages (solid arrows) exchanged between the mobility management modules when a new Mobile Node (MN) first arrives at the WOBAN. These steps are:
(1-5) The MN initially attaches to an AP following standard technology-dependent procedures (in this case following the association procedure defined by IEEE 802.11). The finalization of the layer-2 attachment generates a Link_Up event at the $\mathrm{MN}^{1}$, which sends a Router Solicitation message that reaches the access router - i.e. the MAG. In parallel a Link_Up event is also generated at the PoA $\left(A P_{1}\right)$, which is the corresponding end point of the layer- 2 connection. This event is propagated through an MIH_Link_Up indication to the MAG, triggering the registration to the LMA.

(6) This message triggers the MAG at the ONU to send a Proxy Binding Update to the LMA at the OLT, requesting for an IPv6 prefix for this new MN.

(7) The LMA looks up its Binding Cache and creates a new entry for this new MN. The LMA delegates an IPv6 prefix to the MN, conveyed back to the MAG in a Proxy Binding Acknowledgement message.

(8) The MAG then sends a Router Advertisement to inform the MN about the assigned prefix, allowing the MN to configure a valid IPv6 address using standard Stateless Address Autoconfiguration (SLAAC) techniques.

After this process is completed, the MN has network connectivity, and its traffic should be encapsulated on an IPv6in-IPv6 tunnel from/to the MAG to/from the LMA. Such a tunnel between the MAG and the LMA yields us to the first optimization proposed in the integrated PMIPv6-WOBAN architecture.

Optimization no. 1: No need for IPv6 tunneling. In this case, there is no need for an IP-in-IP tunnel between the MAG and the LMA since the connection is one-hop distant. Essentially, all traffic sent by the OLT is received by all ONUs, which just filter out EPON frames destined to other nodes based on the LLID and destination MAC address. In the uplink direction, the same reasoning applies since the traffic sent by a given ONU arrives only at the OLT (never at the other ONUs). This benefit leverages the point-to-multipoint topology of the EPON and applies to the communication between the LMA-MAGs, thus reducing the overhead of the communication.

\section{B. Handover operation in the integrated architecture}

Now, consider the previous MN moves to a new AP, which triggers a handover in the mobile network. Such an action may occur due to mobility reasons (the $\mathrm{MN}$ is moving away of the radio coverage of the AP) or due to network reasons (the current ONU/AP is overloaded and the network decides to move some users to a neighboring ONU/AP). Then, the IEEE 802.21 MIH framework is used to enhance the handoff performance by making it proactive (the so-called makebefore-break approach). The following procedure assumes that the handover decision and target selection is performed by the OLT-LMA, since it has full knowledge about the status and available resources of the WOBAN. Thus:

\footnotetext{
${ }^{1}$ Following the IEEE 802.21, the Link_Up event is generated by the wireless driver when a layer- 2 connection is established on that particular link interface.
} 
$(9,10)$ At some point in time, the link layer at the MN detects poor signal level (or any other suitable metric), triggering a Link_Going_Down event at the MN, which indicates an imminent loss of radio coverage. This message is propagated to the network entity in charge of the mobility management of the MN (e.g., the PoS), in this case the OLT-LMA. Note that this message follows the path $M N \rightarrow A P \rightarrow O N U \rightarrow O L T$, encapsulated as a layer-3 packet (as defined in RFC 5164).

$(11,12)$ Then, the OLT-LMA suggests a list of suitable PoAs to the MN with the MIH_Net_HO_Candidate_Query request message. This is an optimized list since the OLT-LMA contains both the network load status of the PON (collected via MPCP) and the mobility management information about the number of users attached to each AP (thanks to the MAGs). Thus, the OLT-LMA suggests which APs/channels are worth(22-32) scanning, hence reducing the handover operation delay. The MN indicates its preferences to the OLT-LMA using an MIH_Net_HO_Candidate_Query response message.

(13) Optimization no. 2: Optimal Target Network Selection. The OLT-LMA has all the information regarding the traffic load of each ONU and the geographical location of every AP. Hence, the OLT-LMA can make a decision about the best ONU/AP to handover to.

(14-16) Once the most suitable AP is chosen, the OLT-LMA queries the new MAG for its suitability in hosting the moving $\mathrm{MN}$, via the MIH_N2N_HO_Query_Resources request

primitive. Through this message, the OLT-LMA is also able to inform the target ONU $\left(\mathrm{ONU}_{2}\right)$ of the IP address of the ONU currently serving the $\mathrm{MN}\left(O N U_{1}\right)$. This information is later used to optimize the handover (see step 18). Under the assumption that the target MAG accepts the $\mathrm{MN}$, the new MAG would then reply to the LMA with an MIH_N2N_HO_Query_Resources response message.

The next set of steps corresponds to the handover preparation procedure:

(17) Next, the LMA informs the target MAG $\left(O N U_{2}\right)$ about the imminent handover. The $\mathrm{MN}$ will be notified about the target MAG in step 21, once the handover preparation is ready.

(18-21) Optimization no. 3: Data bicasting during handover. At this moment, the LMA and the target MAG $\left(\mathrm{ONU}_{2}\right)$ know that the MN handoff is imminent. In order to avoid packet loss during the handover process, the OLT sends all the MN traffic to both old and new ONUs (bicasting). To enable this optimization, each PMIPv6WOBAN ONU has an associated multicast MAC address (which may be pre-configured or directly derived from its IP address), and thus receive all the broadcast EPON frames destined to that address, or to any other multicast MAC address they are listening to. Then, it is just necessary that $O N U_{2}$ joins the layer-2 multicast group of $O N U_{1}$ (step 18), and the OLT starts sending the MN traffic to the broadcast LLID and the $O N U_{1}$ multicast MAC address (step 20). This way, the MN traffic is received by $O N U_{1}$ and $O N U_{2}$ only, since the other ONUs filter these EPON frames out because of the destination multicast MAC address (Fig. 4, second box of the simplified data frames). Therefore such multicastbased bicasting does not consume any additional bandwidth capacity of the EPON. The bicasting preparation starts when the target MAG $\left(O N U_{2}\right)$ receives the MIH_N2N_HO_Commit request from the LMA, and before it replies back with an MIH_N2N_HO_Commit response (remark that the IP address of $O N U_{1}$ was sent to $O N U_{2}$ in message 14). Finally, once the data bicasting process has started, the LMA informs the MN that it is now able to perform the actual layer- 2 handover to the target AP (message 21).

The following procedure is very similar to the one explained for messages 1 to 8 . Once the layer- 2 connection to the new AP is established, the MN and $A P_{2}$ generates a Link_Up event, which is used by $M A G_{2}$ to trigger the sending of a Proxy Binding Update message (step 28). Since the PMIPv6 handover is now complete, the OLT can stop the bicasting and send the MN's traffic directly to the new serving MAG $\left(\mathrm{ONU}_{2}\right)$, which also stops listening to the multicast MAC address of $O N U_{1}$ (Fig. 4, bottom right box). Note that the MN keeps using the same IPv6 address, despite the change of MAG/AP since it is provided with the same prefix used in the previous attachment by means of a Router Advertisement (step 32). Thus, in both up/downstream directions, PMIPv6 hides all mobility management details to the MN.

$(33,34)$ Finally, once the handover is complete, the MN notifies the PoS (OLT) through the MIH_MN_HO_Complete messages.

Finally, when an MN moves between two APs of the same ONU-MAG, the handover procedure is much simpler. Essentially, the handover may be performed at layer 2 without the need for any PMIPv6 signaling. Also, data bicasting is not necessary since this type of handover involves a single ONUMAG.

\section{DISCUSSION OF PERFORMANCE OPTIMIZATIONS}

The three mobility optimizations proposed in the previous section have a clear impact on the performance operation of the PMIPv6-WOBAN, as noted from the following sections.

\section{A. Bandwidth waste reduction}

Thanks to the first optimization, no IPv6-in-IPv6 tunnel is required between the LMA and the MAGs, which saves 40 bytes per packet (due to the extra IPv6 tunnel header). This accounts for a $5.6 \%$ of bandwidth waste in realistic wireless access scenarios [11] (with an average packet size of 710 bytes), and could even rise to $46.5 \%$ for voice over IP calls where traffic is encoded with the Internet low bit rate codec (iLBC) used by Skype. This accounts for an important amount of bandwidth savings. 


\section{B. Fast handover procedure}

Real tests conducted with an in-house PMIPv6 implementation show an average handoff interruption time of $950 \mathrm{~ms}$ (without layer-2 triggers). Most of this delay can be reduced thanks to the use of MIH link-layer triggers (to send Proxy Binding Update messages), together with the fact that the OLT-LMA tells the MNs which channels to scan for the handover process (second optimization). In-house measurement studies conducted by the authors with a modified version of the ath $9 \mathrm{k}$ driver ${ }^{2}$ that scans only one channel and includes other layer-2 attachment refinements have shown average handover delays of $68.58 \pm 0.76 \mathrm{~ms}$, which is much smaller than the original one-second handover delay of a PMIPv6 architecture without MIH and optimal channel selection. This optimized delay value is given in Table I and shall be used in the validation section IV-E.

\section{Reduced packet loss}

The bicasting (third) optimization proposed in the integrated architecture, where the OLT-LMA multicasts the traffic of a moving MN to both the old and the new ONU-MAGs, allows minimizing packet loss during the handover process. This optimization does not consume any extra bandwidth, since the MN's data traffic is not duplicated but just transmitted once through the PON. This sharply contrasts with other standardized handover-optimization mechanisms that have a suboptimal routing in WOBAN scenarios. For instance, Fast Handovers for Proxy Mobile IPv6 (FPMIPv6) [12] is based on redirecting the $\mathrm{MN}$ traffic during a handover by means of a direct IPv6-in-IPv6 tunnel between the old and new MAGs. However this solution does not fit well in a WOBAN scenario because all packets sent between two MAGs (i.e. ONUs) need to go through the OLT. Therefore, during an FPMIPv6 handover, the MN downstream traffic would have to be sent first from the LMA to the old MAG $\left(O L T \rightarrow O N U_{1}\right)$, which in turn would encapsulate it in an IPv6-in-IPv6 tunnel and send it to the new MAG $\left(O N U_{1} \rightarrow O L T \rightarrow O N U_{2}\right)$. This is clearly suboptimal for a PON scenario.

\section{Handover estimated delay}

Table I shows an estimate ${ }^{3}$ of the different delays involved in the handover decision, preparation and finalization. Following this $\mathrm{Table}^{4}$, the total worst-case delays in each block of Fig. 4 can be estimated as:

i) Initial attachment: $T_{L 2_{h o}}+T_{A P \leftrightarrow O N U}+T_{O N U \rightarrow O L T}+$ $T_{L M A}+T_{O L T \rightarrow O N U}+T_{A P \leftrightarrow O N U}+T_{W L A N} \simeq 75.6$ $\mathrm{ms}$.

ii) Handover decision: $\left(T_{W L A N}+T_{A P \leftrightarrow O N U}+\right.$ $\left.T_{O N U \rightarrow O L T}\right)+\left(T_{O L T \rightarrow O N U}+T_{A P \leftrightarrow O N U}+T_{W L A N}\right)+$ $\left(T_{W L A N}+T_{A P \leftrightarrow O N U}+T_{O N U \rightarrow O L T}\right)+T_{O L T \rightarrow O N U}+$ $T_{O N U \rightarrow O L T} \simeq 19.14 \mathrm{~ms}$.

\footnotetext{
${ }^{2}$ See http://Iinuxwireless.org/en/users/Drivers/ath9k ${ }^{3}$ The PMIPv6 implementation used in our experiments can be found in http://www.openairinterface.org/ openairinterface-proxy-mobile-ipv6-oai-pmipv6

${ }^{4}$ In Table I, $N$ refers to the number of ONUs (typically 32 or 64 ), $T_{g}=$ $1.5 \mu \mathrm{s}$ refers to the guard time, and $\rho$ is the total traffic load in the PON.
}

iii) Handover preparation: $T_{O L T \rightarrow O N U}+T_{O N U \rightarrow O L T}+$ $\left(T_{O L T \rightarrow O N U}+T_{A P \leftrightarrow O N U}+T_{W L A N}\right) \simeq 6.54 \mathrm{~ms}$.

iv) Handover finalization: $T_{L 2_{h o}}+T_{A P \leftrightarrow O N U}+$ $T_{O N U \rightarrow O L T}+T_{O L T \rightarrow O N U}+\left(T_{A P \leftrightarrow O N U}+T_{W L A N}\right)+$ $\left(T_{W L A N}+T_{A P \leftrightarrow O N U}+T_{O N U \rightarrow O L T}\right)+\left(T_{O L T \rightarrow O N U}+\right.$ $\left.T_{A P \leftrightarrow O N U}+T_{W L A N}\right) \simeq 82.78 \mathrm{~ms}$

Without the bicasting optimization, data loss may occur from the beginning of step 22 (layer- 2 connection) to the end of step 30 (Proxy Binding Acknowledgment), since only at this time the target ONU-MAG may forward data to the incoming MN. Essentially, the bicasting flow allows the target ONUMAG to buffer the MN's data until step 30.

\section{E. Validation of the proposed architecture based on simulation}

This section further evaluates the performance improvements achievable by the optimizations proposed before. To do so, the authors have extended the event-based special-purpose simulator developed in [15] with EPON and PMIPv6 modules. The simulator estimates the service disruption time caused during the handover process of an MN that moves between two APs connected to different ONUs. In our scenario, we consider a PON with $N=32$ ONUs employing IPACT, where the distance between a given ONU and the OLT is $5 \mathrm{~km}$ (i.e. $25 \mu \mathrm{s}$ one-way propagation delay). Each of the ONUs is connected to an IEEE 802.11g AP via Fast Ethernet (we consider a negligible propagation delay between AP and ONU and a serialization delay of $82 \mu s / K$ Byte). To simulate the EPON and specifically the IPACT algorithm, the simulator uses a hybrid packet analysis based approach. On one hand, each packet exchanged between the OLT and the studied MNs in downlink is simulated on a packet by packet basis. On the other hand, uplink traffic follows an analytical model, used to compute the average queuing delay of the packets sent by the MNs. For the validation of our proposal, we consider that the EPON operates at medium load levels (30$40 \%$ [16]). Following this, we assume a total offered load in the upstream channel of $30 \%$ (i.e. $\rho=0.3$ ), thus producing an average cycle time of $E\left(T_{\text {cycle }}\right)=\frac{N T_{g}}{(1-\rho)}=0.23 \mathrm{~ms}$ for a guard time of $T_{g}=5 \mu \mathrm{s}$. In IPACT, the cycle time denotes the time elapsed between the beginning of two consecutive transmission windows for the same ONU in the upstream channel. Hence, we have approximated the average queuing delay of a packet arrival at a given ONU as $\frac{3}{2} E\left(T_{\text {cycle }}\right)$, as noted in [14].

Regarding the PMIPv6 implementation, we extended the OLT with basic PMIPv6 functionality by developing a version of the Binding Cache to store the mapping between Proxy Care-of Address and corresponding IP address of the ONU (MAG), extra functionality required to parse the Proxy Binding Update messages and create the Proxy Binding Acknowledgement was also added. A secondary module, performs a mapping between the ONU's IP and MAC addresses, hence removing the need for an IP tunnel as required by the standard PMIPv6 specification. This module also implements the bicasting functionality by replacing the destination unicast MAC address with the multicast one when necessary (after appropriate signaling is received). 
TABLE I

VALUES USED ON THE THEORETICAL AND SIMULATION ANALYSIS

\begin{tabular}{|c|c|c|c|}
\hline Delay & Analytical model & Comment & Values \\
\hline$T_{W L A N}: \mathrm{MN} \leftrightarrow \mathrm{AP}$ & Gaussian (mean \pm std) & WLAN delay, value from in-house experimentation [13] & $1.18 \pm 0.474 \mathrm{~ms}$ \\
\hline$T_{A P \leftrightarrow O N U}: \mathrm{AP} \leftrightarrow \mathrm{ONU}$ & $82 \mu \mathrm{s} /$ KByte & Serialization delay in a $100 \mathrm{Mbps} \mathrm{Et}$ & $0.12 \mathrm{~ms}$ \\
\hline$T_{O N U \rightarrow O L T}: \mathrm{ONU} \rightarrow \mathrm{OLT}$ & $1.5 \frac{N T_{g}}{1-\rho}$ & Worst case upstream PON TDMA [14] & $5 \mathrm{~ms}$ \\
\hline$T_{O L T \rightarrow O N U}:$ OLT $\rightarrow$ ONU & $5 \mu \mathrm{s} / \mathrm{km}+8.2 \mu \mathrm{s} / \mathrm{KByte}$ & Worst case downs & $0.112 \mathrm{~ms}$ \\
\hline$T_{L 2 h_{\circ}}$ : layer-2 Connection (Wireless Link) & Gaussian (mean \pm std) & Value from in-house experimentation [13] & $68.52 \pm 0.76 \mathrm{~ms}$ \\
\hline$T_{L M A}:$ Processing time at LMA & Gaussian $($ mean \pm std $)$ & Value from in-house experimentation [13] & $0.6 \pm 0.2 \mathrm{~ms}$ \\
\hline
\end{tabular}

In the simulation, the OLT sends data to an MN, which at a random time, initiates a handover between two ONUs. The IEEE 802.21 signaling is implemented as successive message exchanges, but we only consider the actual size of the MIHF messages, not their real content. The impact of this signaling on the simulation is, as expected, an extra delay between the starting of the handover process and the subsequent layer- 2 detachment.

To validate the proposal, we have considered three different traffic sources: $i$ ) the case when the OLT transmits Poisson traffic to the $\mathrm{MN}$ at an average bitrate of 1 and $10 \mathrm{Mbps}$ (packet size of 1500 bytes) which considers the case of aggregated traffic with low and high data rate profile respectively; and $i$ ) the case when the MN receives a Skype-like VoIP communication (iLBC codec, 50 packet/sec, 116 bytes/packet). We further assume a total offered load in the downstream direction of $30 \%$ (same as upstream, used to compute the average queuing time). We simulated each step during the handover process with the parameters explained in Table I, whose values are supported by real testbed scenarios (see related references in the table), and we measured the number of lost packets without any of the proposed optimizations (data bicasting, no need for IPv6 tunneling and optimal target selection) in order to quantify the performance gains in terms of packet loss and connectivity disruption time. The only optimization that impacts the packet loss is the data bicasting, which prevents it completely because the data addressed to the $\mathrm{MN}$ is being bicasted and buffered before its actual movement. In the simulation we consider that the handover process delay is reduced to the value of $T_{L 2_{h o}}$ shown in Table I.

Fig. 5 shows the empirical cumulative distribution function (CDF) of the number of lost packets in a WOBAN as the one proposed, without the data bicasting optimization. As shown, this optimization brings important packet loss savings for the three traffic profiles. For example, in the VoIP traffic profile, the number of lost packets shows a median of four packets (Fig. 5(a)), which implies about $80 \mathrm{~ms}$ of conversation disruption. Similar benefits are shown for the other two traffic profiles, since the data bicasting optimization implies no packet loss during the handover. Finally, Fig. 5(b) presents the handover duration CDF. As previously explained, the handover time for all cases is similar, since we assume an optimized layer-2 delay in all scenarios. As shown in Fig. 5(b), the average handover duration corresponds to $87.29 \mathrm{~ms}$, which is in line with the values computed theoretically in section IV-D.

\section{SUMMARY AND DISCUSSION}

Hybrid Wireless-Optical Broadband Access Networks (WOBANs) are a promising technology to provide high-speed wireless access to end-users. This article takes one step further in the integration of wireless-optical technologies by proposing an integrated PMIPv6-WOBAN architecture that simplifies the mobility management of MNs. This architecture maps the PMIPv6 framework and IEEE 802.21 MIH Services into the hierarchical structure of the WOBAN's Passive Optical Network (PON) by collocating the Local Mobility Anchor (LMA) with the Optical Line Terminal (OLT), and the Mobile Access Gateways (MAGs) with the Optical Network Units (ONUs), which controls a set of heterogeneous wireless Access Points (e.g., WiFi and cellular). Such a tight integration enables the optimized use of resources, since the centralized OLT-LMA node is able to combine traffic statistics and user mobility information collected from the ONU-MAGs, and thus may initiate handovers of MNs due to ONU or AP overload. Moreover, the proposed architecture includes a number of optimizations that leverage the particular characteristics of a WOBAN. For instance, the single-hop, point-to-multipoint topology of an Ethernet PON (EPON) avoids the overhead of maintaining tunnels between the LMA and its MAGs; and enables the use of multicast EPON bicasting during handoffs to prevent from packet loss, therefore providing a seamless handover experience. We argue that the complexity of the proposed architecture is moderate since OLT/ONU devices are already full-fledged IP routers, hence adding LMA/MAG functionality would require of a minor software update. Regarding the optimizations considered, only the Optimized Target Selection may incur in high complexity in case it is integrated with the OLT scheduler, since then a smart scheduler, providing higher bandwidth to the ONUs with a higher number of attached users may be implemented, requiring access to the Dynamic Bandwidth Allocation (DBA) table at the OLT.

Finally, this PMIPv6-WOBAN architecture supports other access technologies different from $\mathrm{WiFi}$ on the Localized Mobility Domain (LMD), and therefore a multi-interfaced MN would be able to roam between different technologies or make use of them simultaneously (e.g., to perform traffic offloading, flow mobility or multi-link aggregation).

\section{ACKNOWLEDGMENTS}

The authors would like to acknowledge the support of the EU-funded MEDIEVAL (grant FP7-ICT-2009-5/258053), the CAM-funded Medianet project (under code S-2009/TIC-1468) and the MICINN research grant TIN2010-20136-C03. 


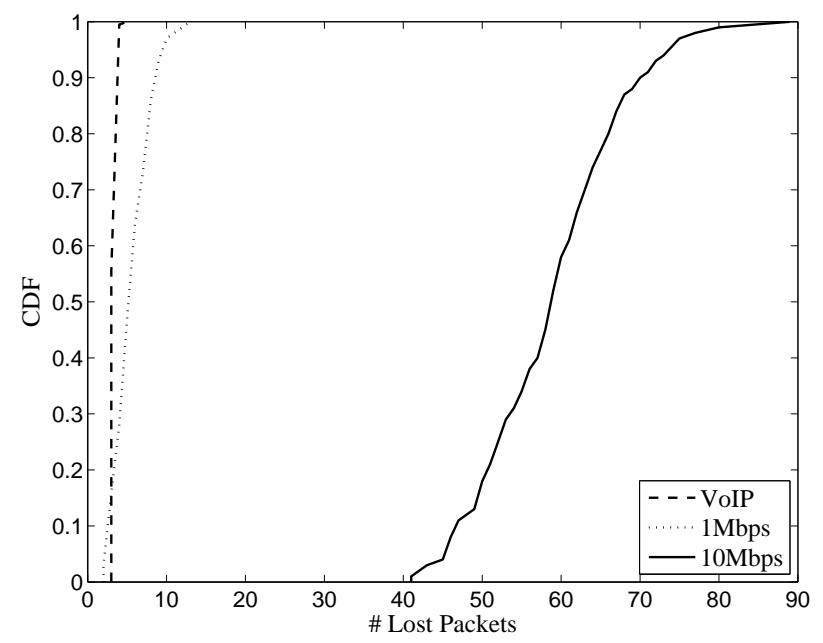

(a)

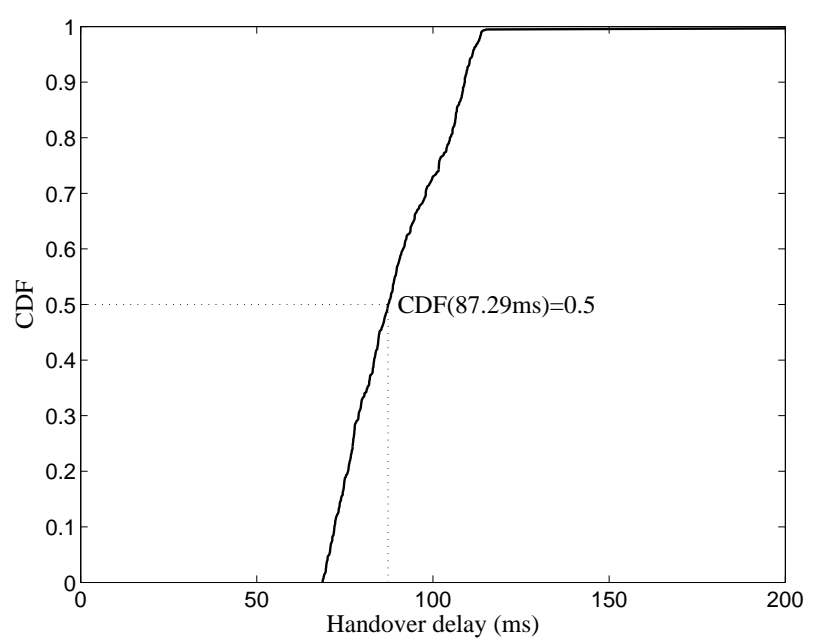

(b)

Fig. 5. Simulation results: (a) CDF of the number of lost packets without optimization and (b) CDF of the optimized handover delay

\section{REFERENCES}

[1] S. Sarkar, S. Dixit, and B. Mukherjee, "Hybrid Wireless-Optical Broadband Access Network (WOBAN): A review of relevant challenges," IEEE/OSA J. Lightwave Technology, vol. 25, no. 11, pp. 3329-3340, Nov. 2007.

[2] P. Chowdhury, B. Mukherjee, S. Sarkar, G. Kramer, and S. Dixit, "Hybrid Wireless-Optical Broadband Access Network (WOBAN): Prototype development and research challenges," IEEE Network, vol. 23, no. 3, pp. 41-48, 2009.

[3] K. Yang, S. Ou, K. Guild, and H.-H. Chen, "Convergence of Ethernet PON and IEEE 802.16 Broadband Access Networks and its QoS-aware dynamic bandwidth allocation scheme," IEEE J. Selected Topics in Communications, vol. 27, no. 2, pp. 101-116, Feb. 2009.

[4] G. Shen, R. Tucker, and C.-J. Chae, "Fixed Mobile Convergence Architectures for Broadband Access: Integration of EPON and WiMAX," IEEE Communications Magazine, vol. 45, no. 8, pp. 44-50, Aug. 2007.

[5] A. Reaz, V. Ramamurthi, S. Sarkar, D. Ghosal, S. Dixit, and B. Mukeherjee, "CaDAR: An efficient routing algorithm for a Wireless-Optical Broadband Access Network," IEE/OSA J. Optical Communications and Networking, vol. 1, no. 5, pp. 392-403, Oct. 2009.

[6] P. Chowdhury, M. Tornatore, S. Sarkar, and B. Mukherjee, "Building a Green Wireless-Optical Broadband Access Network (WOBAN)," IEEE J. Lightwave Technology, vol. 28, no. 16, pp. 2219-2229, Aug. 2010.

[7] S.-H. Lee, J. Kim, and M.-H. Kang, "Performance enhancement in future PON and mobile convergence networks," in 11th International Conference on Advanced Communication Technology (ICACT 2009), vol. 1, Feb. 2009, pp. 233-236.

[8] S. Newaz, Y. Bae, M. Ahsan, and J. Choi, “A study on PMIP deployment over EPON," in 9th International Conference on Optical Internet (COIN 2010), July 2010, pp. 1-3.

[9] G. Kramer, B. Mukherjee, and G. Pesavento, "IPACT: A dynamic protocol for an Ethernet PON (EPON)," IEEE Commumincations Magazine, vol. 40, no. 2, pp. 74-80, 2002.

[10] A. de la Oliva, A. Banchs, I. Soto, T. Melia, and A. Vidal, "An overview of IEEE 802.21: Media-Independent Handover Services," IEEE Wireless Communications, vol. 15, no. 4, pp. 96-103, 2008.

[11] P. Serrano, A. de la Oliva, C. Bernardos, I. Soto, A. Banchs, and A. Azcorra, "A CARMEN mesh experience: deployment and results," in IEEE Workshop on Hot Topics in Mesh Networking, HotMESH'09, 2009

[12] H. Yokota, K. Chowdhury, R. Koodli, B. Patil, and F. Xia, "Fast Handovers for Proxy Mobile IPv6," RFC 5949 (Proposed Standard), Internet Engineering Task Force, Sept. 2010.

[13] A. de la Oliva, I. Soto, M. Calderon, C. J. Bernardos, and M. I. Sanchez, "The costs and benefits of combining different IP mobility standards," Computer Standards \& Interfaces, vol. 35, no. 2, pp. 205 - 217, 2013. [Online]. Available: http: //www. sciencedirect. com/science/article/pii/s092054891200092X
[14] B. Lannoo, L. Verslegers, D. Colle, M. Pickavet, M. Gagnaire, and P. Demeester, "Analytical model for the IPACT dynamic bandwidth allocation algorithm for EPONs," OSA J. Optical Networking, vol. 6, no. 6, pp. 66-688, 2007.

[15] T. Melia, A. de la Oliva, I. Soto, P. Serrano, and R. Aguiar, "Network controlled handovers: challenges and possibilities," Wireless Personal Communications, vol. 43, no. 3, pp. 959-974, 2007.

[16] G. Kramer, B. Mukherjee, and G. Pesavento, "Ethernet pon (EPON): Design and analysis of an optical access network," Photonic Network Communications, vol. 3, no. 3, pp. 307-319, 2001.

M. Isabel Sanchez received a Telecommunication Engineering degree in 2010 and a M.Sc. degree on Telematic Engineering in 2011 both from the University Carlos III of Madrid, where she is currently enrolled in the $\mathrm{PhD}$ program. Since 2010 she is working as Research Assistant at the Institute IMDEA Networks of Madrid, being her main interests vehicular communications and IP mobility in heterogeneous networks.

Manuel Urueña received his M.Sc. degree in Computer Science from Universidad Politcnica de Madrid in 2001 and his Ph.D. degree in Telecommunications from Universidad Carlos III de Madrid in 2005. At present, he is an assistant professor in Telematics Engineering at Universidad Carlos III de Madrid. His research activities range from P2P systems, through load balancing and service discovery protocols, to optical networks.

Antonio de la Oliva received a Telecommunication Engineering degree in 2004, and a PhD in Telematics in 2008, both from the University Carlos III of Madrid (UC3M), where he worked as a research and teaching assistant from 2005 to 2008 and, since then, has worked as an Visiting Professor. His research is focused to mobility in general and specifically to Media Independent Handovers. He has published over 20 scientific papers in prestigious international journals and conferences, and he is also an active contributor and voting member of the IEEE 802.21. 
Jose Alberto Hernandez completed the five-year degree in Telecommunications Engineering at Universidad Carlos III de Madrid (Madrid, Spain) in 2002, and the Ph.D. degree in Computer Science at Loughborough University (Leics, United Kingdom) in 2005. From 2005 to 2009, he was a postdoctoral researcher and teaching assistant at Universidad Autonoma de Madrid. In 2009, he moved to Universidad Carlos III de Madrid, where he became an associate professor. He has published more than 70 articles in both journals and conference in-proceedings in the areas of IP over WDM, Energy Efficient Ethernet, network performance measurements and optimisation.
Carlos J. Bernardos received a telecommunication engineering degree in 2003 and a Ph.D. in telematics in 2006, both from UC3M, where currently he works as an associate professor. From 2003 to 2008 he worked at UC3M as a research and teaching assistant. His current work focuses on IP-based mobile communication protocols and vehicular networks. His Ph.D. thesis focused on route optimization for mobile networks in IPv6 heterogeneous environments. He has actively contributed and held leadership roles in the IETF. He has published over 50 scientific papers in prestigious international journals and conferences and has served as guest editor of IEEE Network. 